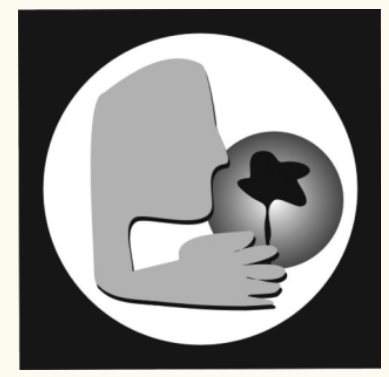

Sustentabilidade e $\mathrm{m}$ Debate

\title{
Adaptación a la Degradación de los Recursos Naturales en la Zona Semiárida Mexicana
}

\begin{abstract}
'Autor para correspondencia. Instituto Nacional de Investigaciones Forestales Agrícolas y Pecuarias (INIFAP) Centro Nacional de Investigación Disciplinaria en Relación Agua-Suelo-Planta Atmósfera (CENID-RASPA). Gómez Palacio Durango México.Email: gonzalez.barrios@inifap.gob.mx
\end{abstract}

${ }^{2}$ Institut de Recherche pour le Développement (IRD) Laboratoire d'Études des Transfers en Hydrologie et Environnement (LTHE). Grenoble France. Email: luc.descroix@ird.fr

\author{
Palabras clave: Cambio \\ climático, Políticas \\ institucionales, \\ Estrategias de adaptación.
}

\section{RESUMEN}

La investigación hidrológica y edafológica en la zona semiárida mexicana ha puesto en evidencia un proceso de erosión hídrica exacerbado por el cambio climático y el cambio de uso del suelo. Los resultados de investigación han inspirado un programa de adaptación exitoso con una visión ambiental, económica y social. El Programa de Servicios Ambientales Hidrológicos (PSAH) consiste en el pago de mano de obra local para la construcción de obras de conservación del suelo y del agua en áreas susceptibles de proporcionar servicios ambientales hidrológicos. Sus resultados cualitativos saltan a la vista: retención del suelo y del agua, mantenimiento de la cobertura vegetal, retención de pobladores en sus comunidades y bienestar social, entre otros. El PSAH como estrategia de adaptación al impacto del cambio climático deberá encontrar eco en otros lugares de México con el fin de replicar sus beneficios en el medio físico y humano de la zona semiárida.

\section{ABSTRACT}

Key-words:

Climate Change, Institutional Policies, Adaptation Strategies.
Hydrology and soil research in the semiarid zone of Mexico highlight an erosion process that is worsened by climate and land use changes. Scientific results have inspired a successful adaptation program with an environmental and socioeconomic scope. The Hydrological Environmental Services Programm.e (PSAH, in Spanish) pays local workers to build soil and water conservation infrastructure in areas with the potential to provide hydrological environmental services. The programm.e's results are still under assessment, but some qualitative results may be already identified: soil and water retention, plant cover recovery, permanence of local people in their comm.unities, and social welfare, among others. The PSAH as a strategy should be echoed in other places of Mexico in order to replicate their benefits on the physical and human environments of the semiarid zone. 


\section{Introducción}

Durante los últimos años la investigación del medio físico y biológico en la zona semiárida mexicana ha permitido poner en evidencia la exacerbación de los procesos de degradación del medio físico y humano por un cambio de uso del suelo desordenado y por los embates de un clima cambiante y cada vez más difícil de prever lo cual dificulta seriamente asegurar la disponibilidad de los recursos hídricos necesarios y las condiciones adecuadas para la producción agropecuaria.

Los habitantes de la zona árida y semiárida rural que ocupa el $53 \%$ del territorio mexicano se transforman así en migrantes potenciales que se dirigen hacia las principales ciudades del país o hacia el extranjero en busca de mejores condiciones de vida que garanticen la sobrevivencia familiar. Esta situación es origen de grandes problemas económicos, de sobrepoblación y de marginación social para los migrantes en las aglomeraciones que los reciben. La degradación de bosques, pastizales y suelos, es una amenaza para el medioambiente y una forma de desertificación de estas regiones semiáridas al modificar el balance hídrico de las laderas (menos retención de agua, más escurrimiento instantáneo y erosión) y de las cuencas (mayor escurrimiento de avenida, menor escurrimiento de base). Se sabe que las aguas infiltradas en las partes altas alimentan los mantos freáticos de las partes bajas. Una disminución de la infiltración aguas arriba afecta de manera importante la recarga de acuíferos y acelera la desertificación de las zonas áridas como sucede en la Comarca Lagunera (BROUSTE, 1996).

La vulnerabilidad del medio físico y humano en la zona semiárida mexicana puede sin embargo ser mitigada y contrarrestada mediante la adopción de actividades organizadas en torno al cuidado del patrimonio natural y al impulso de condiciones que mejoren poco a poco las condiciones de vida de los habitantes de esta zona. Qué hacer? Estudiar las relaciones causa efecto del deterioro del patrimonio natural en esas regiones y proponer las estrategias más pertinentes para contrarrestar los efectos nocivos. Estudiar la forma en que se dan los procesos naturales y productivos para evitar las condiciones adversas al medio biofísico y humano mediante estrategias de adaptación que permitan una mayor protección de los recursos y una mayor estabilidad y seguridad a la población. Tal es el caso de la experiencia del programa de servicios ambientales hidrológicos que se lleva a cabo en el norte de México.

Después de una presentación de la zona de estudio y del programa de servicios ambientales hidrológicos, se abordaran y discutirán los principales resultados de los trabajos puestos en marcha como estrategia de adaptación a la degradación de los recursos naturales en la zona semiárida mexicana.

\section{Materiales y métodos}

La zona de estudio abarca una extensión de $17000 \mathrm{~km} .{ }^{2}$ en la Sierra Madre Occidental del estado de Durango (norte centro de México) y se sitúa entre las coordenadas $24^{\circ}$ y $26.5^{\circ}$ de latitud norte y $106.5^{\circ}$ y $105^{\circ}$ de longitud oeste. La topografía de la zona es accidentada en su parte occidental y ondulada con colinas y valles en su parte oriental, con una altitud que va de los 1.500 a los 2.600 metros sobre el nivel del mar (Figura 1). El clima es de tipo continental semiárido con una estación de lluvias importante de junio a septiembre, una pequeña estación de lluvias de invierno de octubre a enero y una marcada estación seca de febrero a mayo. La pluviometría anual 
en la zona oscila entre 400 y $900 \mathrm{~mm}$. anuales. Los suelos predominantes en la zona de estudio son de tipo Cambisol, Feozem, y Leptosol desarrollados sobre rocas volcano-eruptivas (Ignimbritas, Riolita y Toba). La vegetación natural es de tipo Sabana de altitud con pastizales y acacias: En las partes más altas de montaña (arriba de los $1.700 \mathrm{~m}$.) la vegetación predominante cambia a la de bosque de encino y pino con pastizales.

La hidrografía de esta región está organizada por un sistema de drenaje en cauces de riachuelos, arroyos y ríos que bajan desde las partes más altas de Sierra hacia las planicies endorreicas más bajas y áridas donde desembocaban naturalmente las aguas del río Nazas antes de la construcción de las presas regionales Lázaro Cárdenas y Francisco Zarco.

La zona de estudio tiene una actividad esencialmente agropecuaria con aprovechamiento de las grandes extensiones de pastizales y de tierras cultivadas con forrajes de temporal (maíz, avena) que son aprovechadas para la cría y engorda de ganado bovino para el mercado de carne nacional y norteamericano.

En las últimas décadas, la zona de estudio se ha visto afectada por un intenso cambio de uso de suelo con el propósito de aumentar el uso de los recursos naturales disponibles (agua, suelo, vegetación) para la producción agrícola y ganadera. Esto ha provocado un desequilibrio entre la capacidad de soporte del sistema y la carga real de los sistemas productivos; lo cual ha llevado a una degradación paulatina del patrimonio natural de la zona que se traduce en una importante erosión del suelo y una marcada escasez de agua.

Además de esos problemas de origen productivo y de capacidad de soporte rebasada, la zona de estudio ha sido afectada por el cambio climático que parece desarreglar los periodos lluviosos en términos de intensidad y duración de las lluvias así como en su uniformidad geográfica; el cambio climático tiende a hacer las lluvias de verano mas borrascosas y violentas aumentando con ello la agresividad y fuerza del agua sobre la superficie del suelo desnudo y sobre las plantas vulnerables en germinación o en los estados iniciales del crecimiento vegetal (estadio de plántula). Estas condiciones pueden ser consideradas como de riesgo por significar una perdida potencial del patrimonio natural productivo que se traduce como una descapitalización de suelos y de la vegetación herbácea anual o cultivada de los campesinos y pequeños ganaderos que constituyen el grueso de la población que habita esas regiones marginadas y desfavorecidas tanto climáticamente como económicamente.

\subsection{El programa de servicios ambientales hidrológicos (PSAH)}

En 2005 el gobierno federal mexicano junto con el gobierno estatal de Durango acordaron poner en marcha el programa de servicios ambientales hidrológicos (PSAH) para restaurar el medio biofísico degradado, a través de obras de conservación probadas con éxito para retener el suelo, la humedad y con ello facilitar la instalación de la vegetación; mantener la cantidad y calidad del agua de escurrimiento e inducir la recarga de mantos acuíferos.

En la estructura del programa participaron la Comisión Nacional Forestal (CONAFOR) la Secretaría de Medio mbiente y Recursos Naturales (SEMARNAT), la Procuraduría de Protección al Ambiente (PROFEPA), la Secretaria de Recursos Naturales y Medio Ambiente del Gobierno de Durango (SRNYMA), gobiernos municipales con áreas beneficiadas y prestadores de 
servicios (Unidades de Aprovechamiento Forestal de las Comunidades Forestales, como Tepehuanes) que integraron los expedientes y sometieron al fondo las candidaturas de áreas propicias para realizar las obras de conservación. Estas últimas están debidamente catalogadas en un manual de obras de la institución rectora (CONAFOR, 2004) y deben ser ejecutadas en etapas supervisadas en el terreno por un responsable de la oficina prestadora de servicios e inspeccionadas luego por un perito de campo del programa financiador.

Las principales obras previstas para la zona de estudio fueron:

- Acordonamientos de material vegetal, resultante de las podas de árboles y arbustos, para reducir la velocidad del escurrimiento del agua, aumentar la humedad del suelo, disminuir la erosión y propiciar las condiciones favorables para la regeneración natural.

- Construcción de presas o barreras con materiales diversos (piedra, material vegetal, llantas, costales de tierra) para disminuir la velocidad de escurrimiento del agua y la pérdida de suelo.

- Implementación de obras de control de la erosión en manto y en cárcavas (acomodo de material vegetal muerto en terrazas y laderas, cabeceo de cárcavas y afine de taludes con piedra acomodada).

- Promoción de la conservación de áreas con vegetación natural o reforestada para evitar la pérdida de vegetación y la máxima captación de agua en el suelo.

- Instalación de letreros de información sobre las buenas prácticas en el medio forestal haciendo llamamientos a prestar ayuda para conservar y proteger el bosque evitando talas, desmontes, basura e incendios forestales.

- Instalación de letreros de advertencia sobre la vigilancia permanente del medio forestal por la autoridad ambiental.

El programa de servicios ambientales inició con un fondo y duración determinados que ha servido para apoyar comunidades por un periodo de hasta cinco años (a partir de 2007 el periodo de apoyo se redujo a 3 años) y en superficies beneficiadas con una extensión máxima de 4.000 hectáreas.

A continuación se presentan y discuten los principales resultados de trabajos científicos y del programa de servicios ambientales hidrológicos llevados a cabo en la zona de estudio.

\section{Resultados y discusión}

Los resultados de las investigaciones realizadas sobre la vulnerabilidad del medio físico de la zona de estudio han sido objeto de publicaciones desde 1997 (DESCROIX \& NOUVELOT, 1997; DESCROIX et al., 2000a; GONZÁLEZ BARRIOS et al., 2004). Las investigaciones más recientes centran su atención en la superficie del suelo y su cobertura vegetal ya que tienen un papel determinante en la hidrodinámica del agua de lluvia y en los procesos de infiltración, escurrimiento y erosión (DESCROIX et al., 2008; GONZÁLEZ BARRIOS et al., 2008; GONZÁLEZ BARRIOS et al., 2009b). Los estudios de cambio de uso de suelo ligados a la evolución de estado de la superficie han arrojado resultados contundentes en el sentido de que a menor cobertura vegetal mayor sensibilidad del suelo a los procesos de erosión y de escurrimiento desordenado (DESCROIX et al., 2000b; DESCROIX et al., 2001; DESCROIX et al., 2004). Los resultados de 


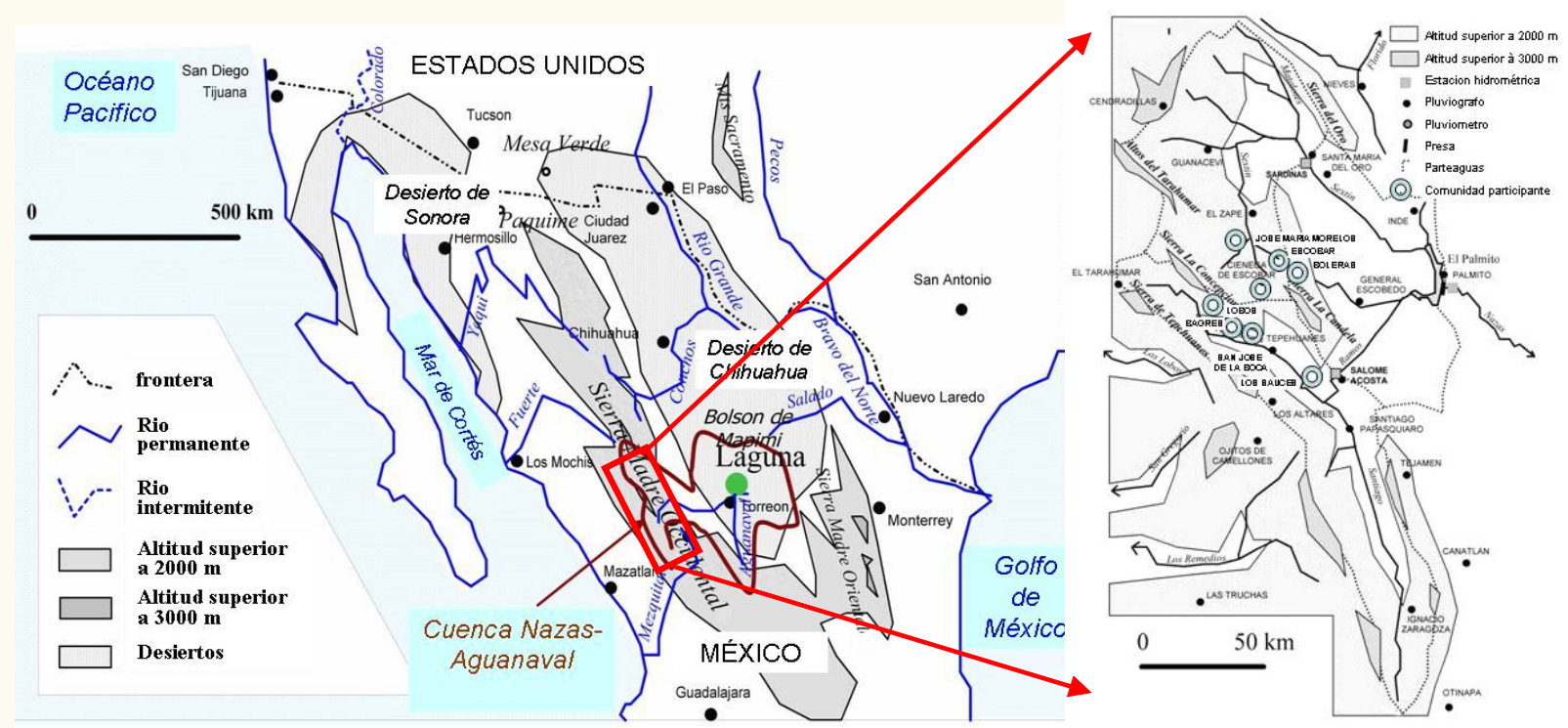

Figura 1. Ubicación de la zona de estudio (izquierda) y comunidades beneficiadas (derecha). (Fuente: L. DESCROIX)

estos trabajos en pequeñas superficies de observación (de 0.2 a $20 \mathrm{~km} .{ }^{2}$ ) revelan una erosión anual en manto de hasta $2.87 \mathrm{Kg}$. de suelo por metro cuadrado que corresponde a un espesor de $1.85 \mathrm{Mm}$.. año ${ }^{-1}$; y una erosión anual en cárcavas de hasta 1.27 toneladas de suelo por metro cuadrado que corresponde a un espesor de $910 \mathrm{Mm}$.. año ${ }^{-1}$ (GONZALEZ BARRIOS, et al., 2005). Estos valores presentan $\sin$ embargo grandes variaciones espacio-temporales en función de la pluviometría anual y del estado de la superficie del suelo (DESCROIX $\&$ POULENARD, 2005). El estudio de la erosión hídrica en manto y en cárcavas ha dado lugar a la propuesta de métodos de medición y seguimiento adaptados al proceso erosivo (DESCROIX et al., 1997b; GONZÁLEZ-BARRIOS et al., 2007;) así como también a la recomendación de medidas paliativas y correctivas a corto y mediano plazo (SÁNCHEZ COHEN et al., 2009a; GONZALEZ BARRIOS, et al., 2009a). Los resultados de investigación han abordado también las formas e intensidades de producción ganadera en las zonas más degradadas donde se observa una carga animal hasta cuatro veces mayor que la capacidad natural de soporte en las superficies forrajeras (VIRAM ONTES, et al., 2004). La erosión en esas áreas de agostadero es causada principalmente por el sobre-pastoreo y el pisoteo del ganado que provoca una fuerte compactación del suelo, modificando la estructura y facilitando el escurrimiento y la pérdida de elementos finos (DESCROIX et al, 2005; GONZALEZ BARRIOS et al., 2010). También se han investigado las condiciones naturales del medio físico que son más susceptibles a la degradación física. Esto es el tipo de suelo, el tamaño de la superficie drenada o área de contribución, la intensidad de la lluvia. Se pudo demostrar que en los suelos con mayor proporción de limos y en las grandes superficies de las sub-cuencas (de 4000 a $7000 \mathrm{~km}^{2}$ ), la erosión en manto puede llegar a ser cien veces mayor que la erosión causada por las cárcavas; esto es sin duda por el efecto de escala de acuerdo a Descroix y colaboradores (2008). Las investigaciones se han orientado además a estudiar las variables hidro-climáticas y sus relaciones con el uso y manejo de los recursos agua, suelo y vegetación. Los efectos 
del clima aún difíciles de pronosticar con exactitud como: heladas, granizadas, inicio y duración del periodo de lluvias, uniformidad geográfica de la pluviometría, temperaturas extremas, han provocado una cascada de consecuencias en el medio físico y humano de esta zona (DESCROIX et al., 1997a; DESCROIX et al., 2002a; SÁNCHEZ COHEN et al., 2008; SÁNCHEZ COHEN et al., 2009b). Los principales resultados de tales investigaciones ponen en evidencia los cambios hidrológicos de las cuencas vertientes más degradadas que se traducen en una alteración del régimen de escurrimiento e infiltración del agua de lluvia (Figura 2) con respuestas hidrológicas más rápidas y violentas que generan escurrimientos de avenida más importantes pero con menor duración; con una disminución correlativa de los escurrimientos de base que revelan una pérdida de capacidad de almacenamiento hídrico del suelo y una mayor vulnerabilidad a los incendios (VIRAMONTES, 2000; DESCROIX et al., 2002b). Los recorridos de campo en la zona de estudio aportan información que confirma la gran incidencia de incendios en áreas de bosque de pino-encino.

Numerosos han sido los artículos publicados señalando las consecuencias hidrológicas de tales condiciones. Sin embargo los efectos de esta degradación del medio físico también se expresan en la población humana ya que al perderse gradualmente el patrimonio natural (suelo, vegetación, recursos hídricos almacenados en forma de humedad del suelo) los habitantes de la región quedan expuestos a las condiciones ambientales cada vez más difíciles de prever para llevar a cabo sus labores productivas y asegurar su sobrevivencia (INARD LOMBARD, 2004).

A partir de 1994 la ley mexicana de tenencia de la tierra permitió la individualización de las tierras colectivas y la división parcelaria del patrimonio natural. Con ello, se hizo más evidente un fraccionamiento territorial y la aparición de una variada gama de condiciones del suelo y de la vegetación que hacen más diversa la calidad de cada propiedad. A casi dos décadas de ese fraccionamiento en algunas comunidades se puede observar que la degradación de algunos sitios colectivos ha sido más intensa que los sitios considerados como privados por la individualización del territorio. Esto recuerda la tragedia de los comuneros (HARDIN, 1968); sin embargo en los sitios de apropiación individual existen también algunos casos de productivismo a ultranza que coinciden con situacio-
1970-1979

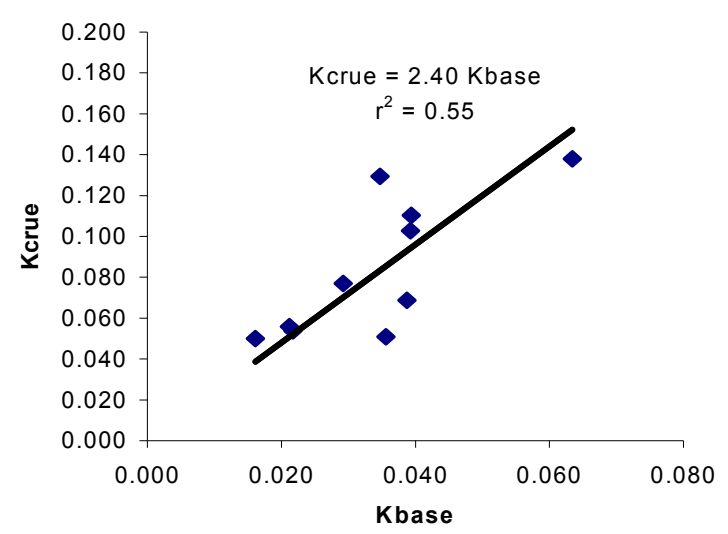

$1990-1998$

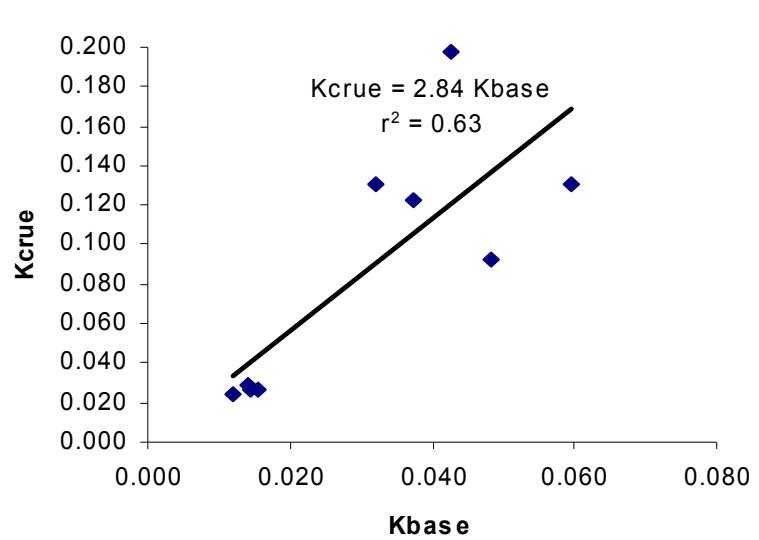

Figura 2. Evolución de la relación entre flujo de crecida (K crue) y tlujo base ( $\mathrm{k}$ base) en la cuenca alta del río Nazas. Periodos de 1970-79 a 1990-98 (Fuente: VIRAMONTES, 2000) 
nes de renta de derechos a terceros. Esto conlleva a la degradación de ese tipo de tenencia de la tierra en la zona. La migración de los propietarios se aceleró ante la falta de poder económico y ante las pobres condiciones del patrimonio natural para seguir produciendo de manera individual o colectiva.

Esta situación y los resultados de los trabajos de investigación correspondientes, inspiraron la instrumentación de un programa de acción con un enfoque integral para combatir el problema de la pérdida del patrimonio natural y humano de la región, la iniciativa fue llamada Programa de Servicios Ambientales Hidrológi$\cos$ (PSAH).

Los resultados del programa de servicios ambientales hidrológicos (PSAH) empezaron de manera modesta (una comunidad beneficiada con 4000 hectáreas en 2006) pero fueron extendiéndose rápidamente (hasta ocho comunidades beneficiadas con 14500 hectáreas en 2009) ante el éxito de respuesta provocado en los habitantes de las comunidades beneficiadas. La mano de obra que fue pagada por dicho programa con un buen salario (equivalente a 30 dólares americanos diarios aproximadamente) atrajo la atención de casi toda la fuerza productiva de las comunidades. Prueba de ello son los registros de numerosos hombres y mujeres de 16 a 70 años que se reclutaron para trabajar. El Cuadro 1 muestra el número de obras realizadas durante el periodo de 2006 a 2009 en las comunidades beneficiadas y el cuadro 2 expone los costos unitarios (en pesos mexicanos) de cada tipo de obra.

Cuadro 1. Obras del PSAH realizadas en Durango durante el periodo 2006 a 2009 (Fuente: Unidad de Servicios Forestales de Tepehuanes)

\begin{tabular}{llllll}
\hline Obra (unidad) & 2006 & 2007 & 2008 & 2009 & Total \\
\hline Brecha corta fuego $(\mathrm{km})$. & 0 & 0 & 15.6 & 56 & 71.6 \\
Cabeceo de cárcavas $\left(\mathrm{m}^{2}\right)$ & 880 & 1250 & 1550 & 2885 & 6565 \\
Cercos (km.) & 52 & 12 & 104 & 0 & 168 \\
Cordones de piedra $\left(\mathrm{m}^{3}\right)$ & 0 & 0 & 350 & 4010 & 4360 \\
Equipos para brigadas anti-incendio & 0 & 9 & 1 & 1 & 11 \\
Letreros informativos & 63 & 8 & 0 & 4 & 75 \\
Mantenimiento de cordones vegetales (ha) & 2380 & 2522 & 1926 & 956 & 7784 \\
Monitoreo de plagas (jornal diario) & 300 & 316 & 16 & 39 & 671 \\
Poda y limpieza preventiva (ha) & 0 & 0 & 30 & 0 & 30 \\
Presa filtrante de llantas (m $\left.{ }^{3}\right)$ & 0 & 0 & 900 & 160 & 1060 \\
Presa filtrante de piedra (m $\left.{ }^{3}\right)$ & 2320 & 2620 & 3530 & 3524 & 11964 \\
Reforestación (ha) & 0 & 0 & 2508 & 0 & 2508 \\
Tratamiento anti-incendio (jornal mensual) & 6 & 36 & 45 & 48 & 135 \\
Zanja trinchera (x 250 ha) & 0 & 0 & 192 & 20 & 212 \\
\hline
\end{tabular}


Cuadro 2. Obras del PSAH realizadas en Durango durante el periodo 2006 a 2009 (Fuente: Unidad de Servicios Forestales de Tepehuanes)

\begin{tabular}{lc}
\hline Obra (unidad) & Costo unidad (pesos mexicanos) \\
\hline Afine de taludes en cárcavas $(200 \mathrm{~m})$ & 136 \\
Bordo de material vegetal acomodado $(100 \mathrm{~m})$ & 169 \\
Cabeceo de cárcava $\left(6 \mathrm{~m}^{2}\right)$ & 69 \\
Cajetes para plantación de árboles $(100)$ & 180 \\
Cortina vegetal rompe viento $(60 \mathrm{~m})$ & 1623 \\
Presa de gaviones $\left(1 \mathrm{~m}^{3}\right)$ & 614 \\
Presa de llantas $\left(1 \mathrm{~m}^{3}\right)$ & 331 \\
Presa de mampostería $\left(1 \mathrm{~m}^{3}\right)$ & 922 \\
Presa de piedra acomodada $\left(1 \mathrm{~m}^{3}\right)$ & 423 \\
Presa de ramas de árbol $(2 \mathrm{~m} \mathrm{x} 1 \mathrm{~m})$ & 74 \\
Presa de sacos de arena $\left(1 \mathrm{~m}^{3}\right)$ & 259 \\
Presa de geocostales $\left(1 \mathrm{~m}^{3}\right)$ & 429 \\
Superficie reforestada $\left(1 \mathrm{ha}^{3}\right)$ & 895 \\
Terraza de formación sucesiva $(100 \mathrm{~m})$ & 443 \\
Zanja bordo (100 m) & 388 \\
\hline
\end{tabular}

La Figura 3 presenta las imágenes de algunas de las obras de conservación así como la respuesta de los trabajadores organizados en cuadrillas de trabajo que ayudan a recuperar paulatinamente el patrimonio natural que se estaba perdiendo. La evaluación de los efectos de las obras de conservación del suelo y el agua es tema de actividades actuales pero sus excelentes resultados cualitativos son evidentes desde la primera temporada de lluvias.

En 2010 el PSAH llegó a su fin en muchas comunidades piloto con resultados satisfactorios que saltan a la vista, retención de suelo, aumento de la vegetación, restauración paulatina del ecosistema y uniformización o restablecimiento de áreas fraccionadas, además del arraigo de la población a sus comunidades de origen. Sin embargo el programa debe dar lugar a una fase diferente y autofinanciable de los trabajos de conservación para poder mantener las superficies restauradas y expandirlas a las áreas vecinas no beneficiadas por el programa para asegurar sus servicios ambientales hidrológicos. Ello significa la necesidad de más mano de obra pero también significa la necesidad de recursos económicos para llevarlas a acabo. 


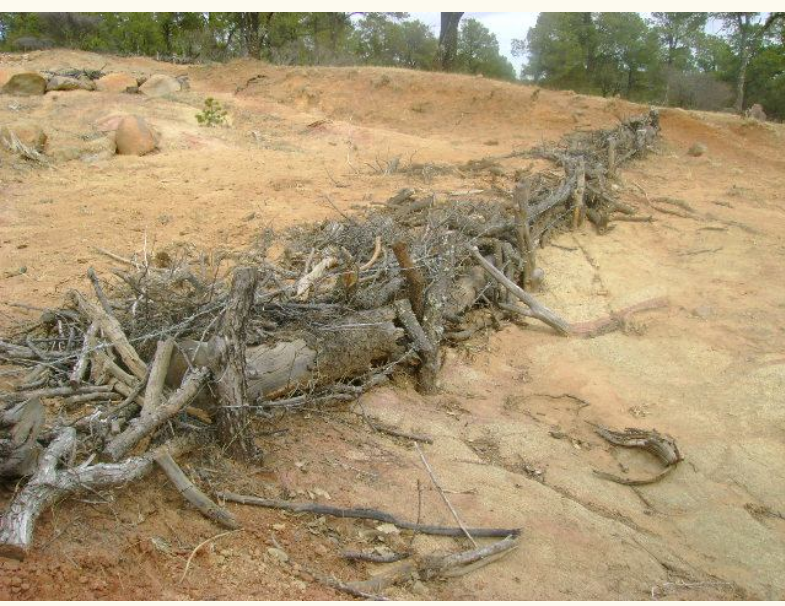

Acordonamiento de material vegetal

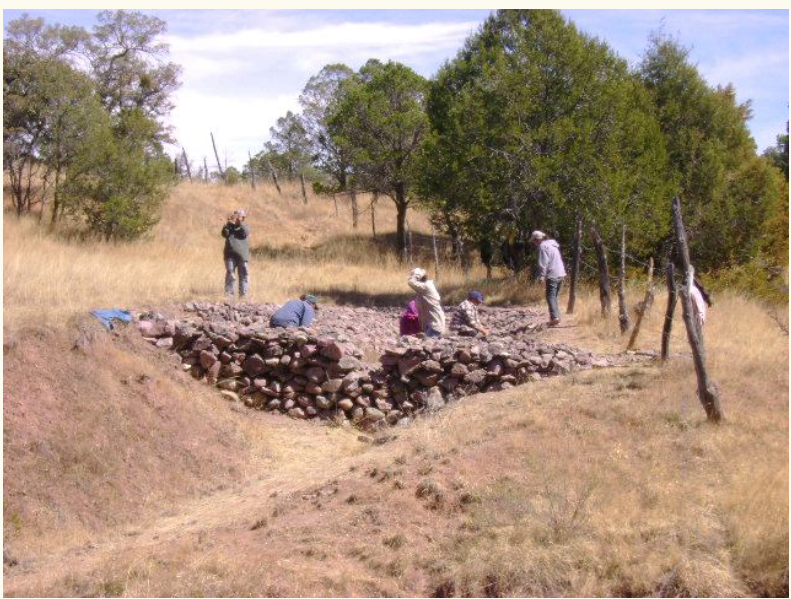

Construcción de presas de piedras

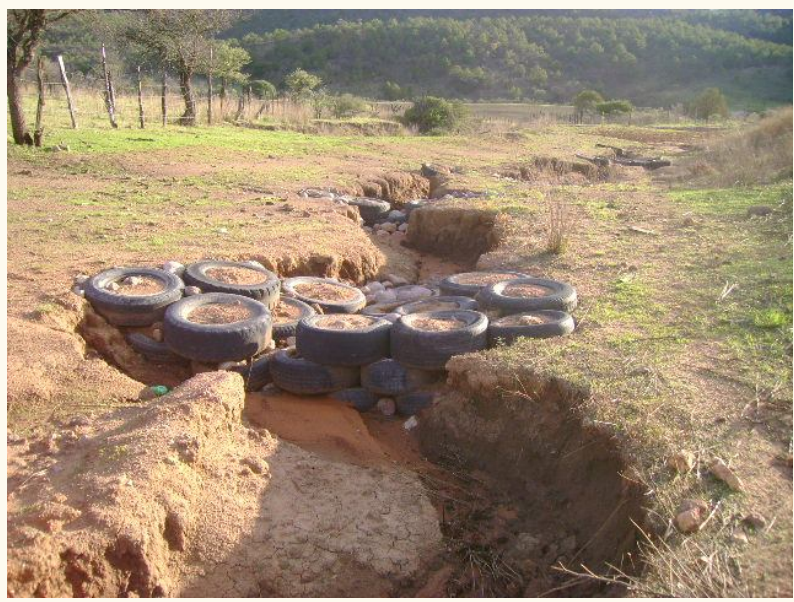

Construcción de presas de llantas

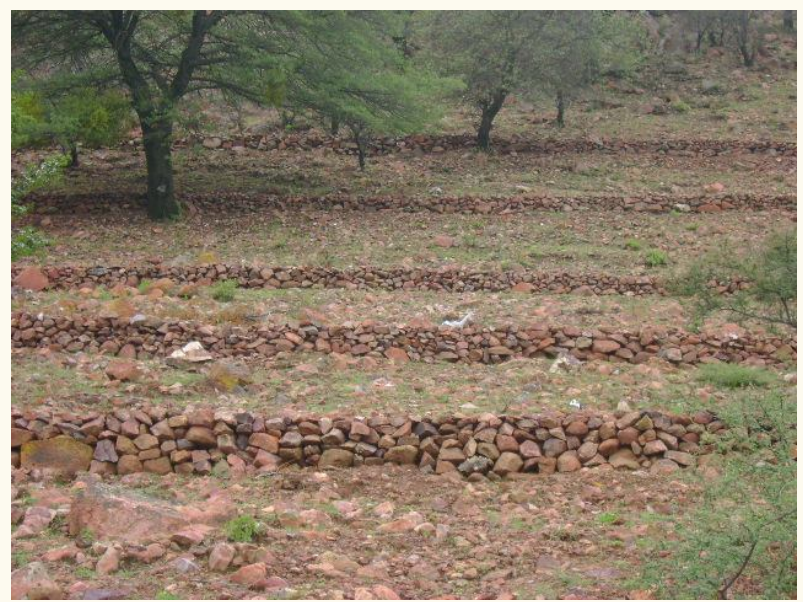

Cordones de piedra acomodada en laderas

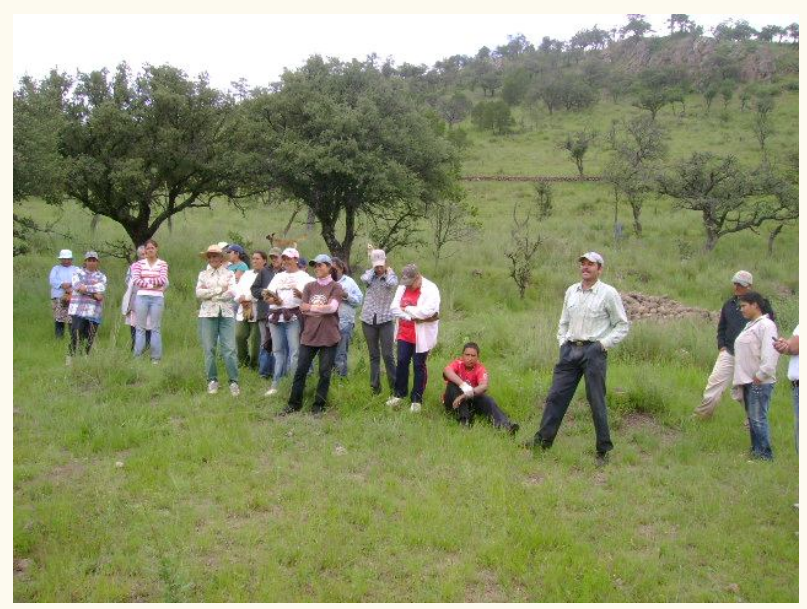

Cuadrilla de trabajo

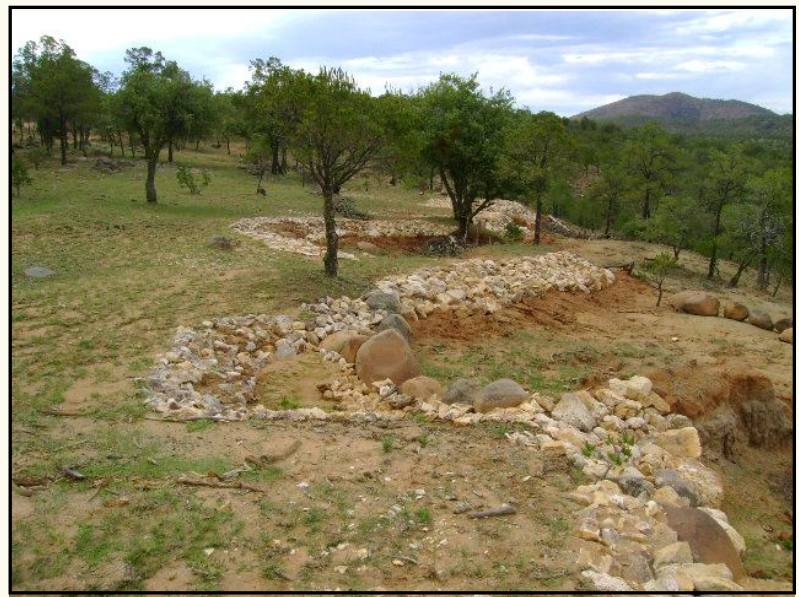

Cabeceo de cárcavas y afine de taludes

Figura 3. Obras realizadas por el Programa de Servicios Ambientales Hidrológicos

(Fuente: J.L. GONZÁLEZ BARRIOS) 


\section{Sustentabilidade em Debate}

Es por ello que los resultados del programa de servicios ambientales hidrológicos se deben dar a conocer a los habitantes de la región árida situada aguas abajo que se beneficia de los servicios hidrológicos realizados en la zona semiárida más alta; que al conservar en buen estado su suelo y su vegetación seguirá generando el agua de escurrimiento y de infiltración de buena calidad para mantener la sobrevivencia y los procesos productivos de esa importante región árida vecina. En 2010 se lanzó el proyecto de pago voluntario por servicios ambientales hidrológicos que pretende sensibilizar a la población beneficiada aguas abajo y que podrá contribuir a la perennización de los trabajos de conservación aguas arriba, en la zona semiárida. El pago voluntario de servicios entró en fase de prueba con el beneficio fiscal de la deducción de impuestos para quien aporta una ayuda económica, sin embargo la mayor recaudación no se espera que venga de las industrias o grandes grupos económicos fuertes sino del grueso de la población que desde su modesta participación económica individual podrá sumar las mayores aportaciones monetarias, como sucede ya en otra región del norte de México (Sierra de Zapalinamé y habitantes de la ciudad de Saltillo) para dar mayor viabilidad a este proyecto (Canales, 2009). Es pues importante y necesario sensibilizar a los habitantes de la región árida aguas abajo para que sus aportaciones económicas voluntarias aseguren el pago de mano de obra suficiente y la ampliación gradual de superficies protegidas hasta llegar a cubrir el 100 $\%$ de lo requerido y restaurar con ello de manera completa el patrimonio natural de esta zona. Solo así podrá completarse el esquema planteado desde el principio y realizar con él la conservación y restauración de esta zona semiárida mexicana como una exitosa adaptación a los impactos adversos del clima y de las actividades humanas.

\section{Conclusión}

A la luz de los resultados, el programa de servicios ambientales hidrológicos se perfila como una primera etapa de la solución a mediano plazo con una visión más integral del problema y de sus soluciones; por un lado la vulnerabilidad natural del medio biofísico a ciertas actividades productivas que requieren sin embargo de mayor control para evitar que sean abusivas; y por otro lado la incertidumbre y la variabilidad del clima que es aún difícil de pronosticar con exactitud pero que es cada vez mas estudiada y analizada por grupos científicos especializados.

El programa de servicios ambientales hidrológicos es un programa piloto importante pero aún insuficiente para restaurar todas las áreas de interés hidrológico, ya que tiene una duración determinada al final de la cual los gestores de los "fondos semilla" deben facilitar la organización de las comunidades beneficiadas para seguir conservando y ampliar las superficies protegidas pero con la ayuda de la sociedad local o de aguas abajo mediante la concientización sobre la importancia de la conservación del medio natural y los beneficios que reditúa en términos de suministro de agua de buena calidad para el consumo ganadero, agrícola y humano, así como para elevar la seguridad hidrológica en el área mediante la reducción de la pérdida del suelo y el mantenimiento de la calidad del agua, la conservación de manantiales y el mantenimiento de la capacidad de recarga de mantos acuíferos, el mantenimiento de escurrimiento de base en época seca, y la prevención de inundaciones o deslizamientos de suelo mediante la delimitación y conservación de áreas de amortiguamiento. El 
reto es evitar la degradación de esa zona semiárida de montaña ubicada en la Sierra Madre Occidental que representa el almacén natural del agua de las lluvias; y evitar la desertificación de las zonas áridas ubicadas aguas abajo que dependen completamente de las aguas foráneas tanto superficiales como subterráneas, para su sobrevivencia y para sus actividades económicas.

El PSAH deberá ayudar entonces a la adopción del pago voluntario por servicios ambientales que permita perennizar a mediano plazo las labores de restauración del ecosistema y el empleo de mano de obra local que arraigue a la población a sus comunidades de origen y facilite con ello una rápida recuperación del patrimonio natural y productivo.

Estas medidas se lograran seguramente con el apoyo sostenido del grueso de la población de quien se esperan las aportaciones económicas más significativas.

La adaptación de la población a los impactos del cambio climático pasa irremediablemente por la restauración del medio biofísico y por el arraigo de la población a sus lugares de origen lo cual da lugar a otras opciones de conservación igualmente viables y explorables que valdrían la pena ser probadas para beneficiar un mayor número de hectáreas de esta zona semiárida. Así por ejemplo los programas de captura de carbono, de protección de la diversidad biológica y de conservación de sitios con belleza escénica son también opciones importantes por valorar en las comunidades sensibilizadas para garantizar el buen estado de la zona semiárida mexicana.

Medidas de control administrativo serán sin embargo obligatorias para impedir la politización de las estructuras de estos programas y para que los recursos recabados de la población beneficiaria sean manejados con absoluta transparencia y honestidad, mostrando en todo momento los cos- tos de las obras de conservación realizadas y los beneficios ambientales y sociales producidos.

Las nuevas propuestas de perennización y de réplica de las obras de conservación deben ser acompañadas de una apertura hacia los mercados internacionales (bonos de carbono, pago de servicios ambientales hidrológicos, protección de áreas biodiversas...) que permitan restaurar los ecosistemas semiáridos de México y de otras regiones del mundo.

\section{Agradecimientos}

Los autores desean expresar su agradecimiento a todas las instituciones que hicieron posible este trabajo en particular al CONACYT al INIFAP al IRD y a los habitantes de las comunidades de Boleras, Escobar y Anexas, Ciénega de Escobar, San José de la Boca, Tepehuanes, así como a las unidades de servicios forestales de Tepehuanes por su amabilidad y disponibilidad en el trabajo de campo de esta investigación.

\section{Referencias}

BROUSTE, L. Hydrochimie et géochimie isotopique de la nappe phréatique de la Comarca Lagunera (Nord-Mexique). Thèse doctorale Université de Paris 11 Orsay, Paris, France, 1997

CANALES, E. Servicios ambientales, análisis de dos casos. En: Memorias del $7^{\circ}$ encuentro sobre biodiversidad y desarrollo sostenible en la laguna. ITESM Campus Laguna. Torreón Coahuila, México, 2009

DESCROIX, L.; NOUVELOT, J.F. Escurrimiento y erosión en la Sierra Madre Occidental. Folleto científico No. 7 CENID- 
RASPA, Gómez Palacio, Durango, México, 1997 DESCROIX, L.; NOUVELOT, J.F.; ESTRADA, J. Geografía de las lluvias en una cuenca del Norte de México: Regionalización de las precipitaciones en la Región hidrológica 36. Folleto científico No. 8 CENID-RASPA, Gómez Palacio, Durango, México, 1997a

DESCROIX, L. ; GUÉDEZ, P.Y. ; POULENAD, J. Méthodes de mesure de l'érosion actuelle: applications dans les Préalpes du Sud (France) et la Sierra Madre Occidentale (Mexique).Bull. du Réseau Erosion No.17, 239-254, 1997b

DESCROIX, L. ; VIRAMONTES, D. ; ANAYA, E. ; POULENARD, J. ; GONZÁLEZBARRIOS, J.L. L'impact du surpaturage et du deboisement sur l'erosion des sols dans la Sierra Madre Occidentale. Bull. du Réseau Erosion No.20, 218-231, 2000a

DESCROIX, L. ; DIGONNET, S. ; GONZÁLEZ-BARRIOS, J.L. ; VIRAMONTES, D. ; BOLLERY, A. Local factors controlling gully or areal erosion in the Sierra Madre Occidental (Northern Mexico). In: Abstracts of the International Symposium on gully erosion under global change. European Soc. for Soil Conservation, Catholic Univ. of Louvain, April 2000. Brussels, Belgium. 2000b

DESCROIX, L.; VIRAMONTES, D.; VAUCLIN, M.; GONZÁLEZ-BARRIOS, J.L.; ESTEVES M. Influence of soil surface features and vegetation on runoff and erosion in the Western Sierra Madre (Durango, Northwest Mexico). CATENA Vol. 43(2),115-135, 2001

DESCROIX, L.; NOUVELOT J. F.; VAUCLIN, M. Evaluation of an antecedent precipitation index to model runoff yield in the Western Sierra Madre (North-west Mexico). J. of Hydrology 263,114-130, (2002a)

DESCROIX, L.; GONZÁLEZ BARRIOS, J. L.;
VANDERVAERE J.P.; VIRAMONTES, D.; BOLLERY, A. An experimental analysis of hydrodynamic behavior on soils and hillslopes in a subtropical mountainous environment (Western Sierra Madre, Mexico). Journal of Hydrology 266(2000),1-14, 2002b

DESCROIX, L.; GONZALEZ BARRIOS, J.L.; VIRAMONTES D.; ESTEVES, M. Gully erosion and land use changes on the hillslopes of the western sierra madre. In: Gully erosion under global change (edited by $\mathrm{Y}$. Li, J. POESEN, \& C. VALENTIN) Chapter 12, 163174. $1^{\text {st }}$ Edition Sichuan Science and Technology Press, Chengdu, China, 2004

DESCROIX, L. ; POULENAD, J. Les formes d'érosion dans la Sierra Madre Occidentale (Nord Ouest du Meique). Bull. Labo.Rhod. Géomorph. Lyon, France. 33-34,1-19, 2005

DESCROIX, L. ; BESNIER, A-L. ; GAUTHIER. E.; AMOGU, O.; VIRAMONTES, D.; GONZALEZ BARRIOS, J. L. Sediment budget as evidence of land-use changes in montaineous areas: two stages of evolution In: Sediment Budgets 2 (Ed. by HOROWITZ, A.J. and Des E. WALLING) 262-270. IAHS Publ. 292, IAHS Press. Wallingford, UK. 2005 DESCROIX, L.; GONZÁLEZ BARRIOS, J.L.; VIRAMONTES, D.; POULENARD. J.; ANAYA, E.; ESTEVES, M.; ESTRADA, J. Gully and sheet erosion on subtropical mountain slopes: Their respective roles and the scale effect. CATENA 72, 325-339, 2008 HARDIN G. The tragedy of the commons. Science 162:1243-1248, 1968

INARD LOMBARD, B. Una Montaña en vías del abandono? En: La Sierra Madre Occidental, una fuente de agua amenazada (Ed. by L. DESCROIX, J.L. GONZÁLEZ BARRIOS \& J. ESTRADA) Capitulo 2, 65-83. Ediciones INIFAP-IRD, Gómez Palacio, 
Durango, México. 2004

GONZÁLEZ BARRIOS, J. L.; DESCROIX, L.; VIRAMONTES, D.; POULENARD, J.; PLENECASSAGNE, A.; MACIAS, L.; BOYER, CH.; BOLLERY, A.; JASSO IBARRA, R. Condiciones que favorecen la erosión y el escurrimiento en manto. En: La Sierra Madre Occidental, una fuente de agua amenazada (Ed. by L. DESCROIX, J.L. GONZÁLEZ BARRIOS \& J. ESTRADA) Capitulo 7, 161178. Ediciones INIFAP-IRD, Gómez Palacio, Durango, México. 2004

GONZÁLEZ BARRIOS, J. L.; ESTRADA AVALOS, J.; GONZÁLEZ CERVANTES, G.; JASSO, R.; SÁNCHEZ COHEN, I.; DESCROIX L. Erosión en cárcavas en la cuenca alta del río Nazas, impacto del cambio de uso del suelo. In: Memorias del XIII Congreso Nacional de Irrigación, Mesa I. Hidrología y Manejo integrado de cuencas. Acapulco, México, 2005

GONZÁLEZ BARRIOS, J.L.; ESTRADA ÁVALOS, J.; JASSO IBARRA, R.; RIVERA GONZÁLEZ, M.; GONZÁLEZ CERVANTES, G. Metodología de observación de cárcavas de erosión en una cuenca hidrológica experimental. Agrofaz Vol. 7 (3), 115-123, 2007

GONZÁLEZ BARRIOS, J. L.; MUÑOZ VILLALOBOS, J. A.; VALENZUELA NÚÑEZ, L. M.; RIVERA GONZÁLEZ, M.; ESTRADAAVALOS, J.; TRUCÍOS CACIANO,

\section{R. Erosión hídrica de suelo y carbono orgánico} en la cuenca alta del Nazas. En: Memorias de la XX Semana Internacional de Agronomía FAZUJED. Noviembre 2008, 458-464. Gómez Palacio Durango, México, 2008

GONZÁLEZ BARRIOS, J.L.; TRUCÍOS CACIANO, R.; MOJICA GUERRERO, A. S.; VALENZUELA NÚÑEZ, L.M. Servicios ambientales hidrológicos en la Sierra de la
Candela Durango, México. Revista Chapingo Serie Zonas Áridas Vol. 8 (1), 43-48, 2009a GONZÁLEZ BARRIOS, J. L.; VANDERVAERE, J. P.; DESCROIX, L.; SÁNCHEZ COHEN, I. Impacto del cambio de uso de suelo en la hidrodinámica superficial de una cuenca receptora de agua. En: Memorias de la Reunión Temática del Agua CONACYT. Cocoyoc Morelos, México, 2009b GONZÁLEZ BARRIOS, J. L.; GONZALEZ CERVANTES, G.; SÁNCHEZ COHEN, I.; LOPEZ SANTOS, A.; VALENZUELA NUÑEZ, L.M.; MUÑOZ VILLALOBOS, J.A.; VELASQUEZ VALLE, M. Ganadería extensiva y alteración de procesos hidroedafológicos en la cuenca alta del río Nazas. En: Memorias de la Reunión Nacional de Innovación forestal y agroalimentaria en México. Campeche, México, 2010

VIRAMONTES, D. Comportement hydrodynamique des milieux dans la Sierra Madre Occidentale: causes et conséquences de leur évolution. Thèse doctorale Université Joseph Fourier de Grenoble. Institut de Géographie Alpine, Grenoble, France, 2000 VIRAMONTES, D.; ANAYA, E.; GARCÍA, C.; POULENARD, J.; BARRAL, H.; MACÍAS, L.; RODRÍGUEZ CAMARILLO M.G. Demasiado ganado y demasiados leñadores: una economía minera. En: La Sierra Madre Occidental, una fuente de agua amenazada ( $\mathrm{Ed}$. by $\mathrm{L}$. DESCROIX, J.L. GONZÁLEZ BARRIOS \& J. ESTRADA) Capitulo 8, 183-193. Ediciones INIFAP-IRD, Gómez Palacio, Durango, México, 2004

SÁNCHEZ COHEN, I.; DÍAZ PADILLA, G.; OJEDA, W.; CHEBHOUNI, G.; ORONA, I.; VILLANUEVA, J.; GONZALEZ BARRIOS, J.L.; GONZALEZ CERVANTES G. Variabilidad climática en México: algunos 
impactos hidrológicos, sociales y económicos. Ingeniería hidráulica en México. Vol. XXIII (4), 5-24, 2008

SÁNCHEZ COHEN, I.; CERANO PAREDES J.; VILLANUEVA, J.; ESTRADA, J.; GONZALEZ BARRIOS, J.L.; CORNEJO, E. Evaluación de alternativas para la conservación de la cubierta vegetal en la parte alta de la cuenca Nazas-Aguanaval mediante el sistema de ayuda para la toma de decisiones (DSS). Agrofaz Vol.9 (5), 55-62, 2009a

SÁNCHEZ COHEN, I.; GONZÁLEZ BARRIOS, J. L.; DÍAZ PADILLA, G. ;VELÁSQUEZ VALLE, M. Cambio climático y vulnerabilidad ecológica: Impacto en las variables hidrológicas de las cuencas. En: Manejo Comparado de Cuencas Hidrológicas; Incertidumbre Climática, Vulnerabilidad Ecológica y Conflicto Social (Comp. by J.L. GONZÁLEZ BARRIOS e I. SÁNCHEZ COHEN) 23-45. Ediciones SMCS CONACYT RETAC INIFAP RNIAS. Torreón Coahuila, México, 2009b

\section{Información de los autores:}

José Luis GONZALEZ BARRIOS es Biólogo, doctorado en hidrología-edafología. Investigador del Instituto Nacional de Investigaciones Forestales Agrícolas y Pecuarias (INIFAP) adscrito al programa de manejo de cuencas hidrológicas en el Centro Nacional de Investigación Disciplinaria en Relación Agua-Suelo-Planta Atmósfera (CENID-RASPA) de Gómez Palacio Durango México. Email: gonzalez.barrios@inifap.gob.mx Luc DESCROIX es Geógrafo doctorado en hidrología. Investigador del Institut de Recherche pour le Développement (IRD) adscrito al Laboratoire d'Études des Transfers en Hydrologie et Environnement (LTHE) de Grenoble France. Email: luc.DESCROIX@ird.fr 